\title{
XII.
}

Aus dem pharmakologischen Institut zu Heidelberg.

\section{Ueber Diurese.}

\author{
III. Mittheilung: \\ Ueber die Beziehungen der Plethora zur Diurese. \\ Von
}

Dr. R. Magnus,

Privatdocent und Assistent des Instituts.

(Mit 2 Curven.)

Wenn zur Erzeugung von Diurese starke Salzlösungen intravenös eingeführt werden, so tritt Salz ans dem Blut ins Gewebe, dafür aber strömt Gewebswasser ins Blut, die Gesammtblutmenge nimmt zu, es kommt zur bydrämischen Plethora. Anhänger der Ludwig'schen Lehre, in neuerer Zeit besonders Starling, leiten nun die Salzdiurese von dieser hydrämischen Plethora ab, welohe zu einer Steigerung capillaren Druckes und dadurch zu stärkerer Harnabscheidung führen soll. Daneben aber komme es noch zu einer activen Dilatation der Nierengefässe, wodurch ebenfalls der capillare Druck in der Niere steigt. Sehen wir von letzterem Moment vorläufig $a b$, so wird also der Hauptwerth auf die entstandene Plethora gelegt und daraus mechaniseh die Harnfluth erklärt.

Im Gegensatz hierzn war ioh in einer vor Kurzem ersehienenen Arbeit 1) zu dem Resultat gekommen, dass die Steigerung des capillaren Druckes nicht für die erste Ursache der Diurese gehalten werden könne, sondern dass die Aenderung der Blutbeschaffenheit, die Zunahme von einem Salze oder von Wasser im Blute oder von beiden zusammen im Wesentlicben die gesteigerte Harnabsonderung bedinge. Die Niere arbeitet dann solange, bis das Plus an dem betreffenden Blutbestandtheil aus der Gefässbahn verschwindet. Nach dieser Anschauung ist es also die Zusammensetzung des Blutes,

1) R. Magrus, Ueber Diurese. II. Mitth. Archiv f. experiment. Pathol. $u$. Pharmakol. Bd. XLIV. S. 396. 1900. 
anf welche die Niere eingestellt ist. Es muss demnach von der entstandenen hydrämischen Plethora nicht die Plethora, sondern die Hydrämie bezw. der vermehrte Gehalt an einem Salze für die Diurese verantwortlich gemacht werden. Die Kreislaufsänderung kann nicht die Rolle der einzigen bedingenden Ursache für die Zunahme der Nierenthätigkeit spielen. Es liess sich zeigen, dass Diurese unabhängig von Aenderungen des arteriellen und venösen Druckes, sowie von. Schwankungen des Onkometerstandes eintrat.

Damit war jedoch nicht gesagt, dass die Aenderungen des Kreislaufes durch die Niere jeder Bedeutung für die Entstehung der Salzdiurese entbehren. Es wäre sehr gut möglich, dass sie die Stärke der Harnabsonderung in wesentlicher Weise unterstïtzen. Demnach ist zu untersuchen, wie gross der Antheil ist, den die reine Aenderung des Kreislaufes in den Nieren am Zustandekommen der Salzdiurese hat.

Lassen wir hierbei eine active dilatatorische Betheiligung der Nierengefässe zunächst ausser Betracht - diese wird in der IV. Mittheilung ausführlich besprochen werden -, so ergiebt sich hieraus die Aufgabe, festzustellen, ob es gelingt, durch Erzeugung einer wahren Plethora den eapillaren Druek zu steigern, und ob dann unter diesem gesteigerten Capillardruck Diurese auftritt. Natürlich musste dabei, um diese Einflüsse ganz isolirt untersuehen zu können, jede Aenderung der Blutbeschaffenheit vermieden werden. Das einzige Mittel, dass zu diesem Ziele führte, schien die Einführung von Blut derselben Thierart zu sein, also Transfusion. Hierbei wird, bestimmte nachher zu besprechende Bedingungen vorausgesetzt, die Zusammensetzung des Blutes nicht geändert. Dagegen ergeben sich dann Kreislaufsänderungen, welche sich mit den nach Salzwasserinfusion beobachteten in Parallele setzen lassen.

Man erhält also Aenderung der Nierencirculation obne Aenderung der Blutbeschaffenheit!

Es erheben sich nun folgende Fragen:

1. Vermögen derartige Kreislaufsänderungen ïberhaupt gesteigerte Harnsecretion zu erzeugen?

2. Wenn dieses der Fall ist, ist dann die hervorgerufene Diurese von derselben Grössenordnung wie bei der Salzdiurese?

Zur Beantwortung dieser Fragen wurden nachstehend geschilderte Versuche angestellt. Der Gang der Untersuchung war vorgezeichnet. Er hatte sich, was Feststellung der Diurese und der Kreislaufsänderungen anlangt, an den in der II. Mittheilung besprochenen Untersuchungsgang anzuschliessen. 
Die Litteratur über die Harnabscheidung nach Transfusion ist spärlich. Nur Ponfickl) berichtet über einige einschlägige Versuche. Hunde zeigten nach Infusion von defibrinirtem Blut keine wesentliche Vermehrung der Harnmenge. Ein Hund, dem Hundeblut direct transfundirt wurde, zeigte ebenfalls "ziemlich sparsame Harnsecretion. Genauere Messungen wurden nicht ausgefüht. Es war deshalb die Wirkung der Transfusion auf die Harnabscheidung noch festzustellen.

Zur Ueberfibrung von Blut auf die Versuchsthiere wurde die directe Transfusion undefibrinirten Blutes von Thier zu Thier angewendet und von der Einspritzung defibrinirten Blutes ganz abgeseben. Schon Lesser ${ }^{2)}$ konnte im Laboratorium von Ludwig feststellen, dass defibrinirtes Blut sich in der Blutbahn anders verhält wie unverändertes Blut und v. Schröder ${ }^{3}$ ) wies darauf hin, dass defibrinirtes Blut bei diuretischen Versuchen nicht als normales anzusehen sei. Auch Schwarz ${ }^{4}$ sah bei Kaninchen Herzstillstand auftreten. Bei den folgenden Experimenten, bei denen es darauf ankam, die Blutbeschaffenheit möglichst wenig zu ändern, schien die directe Transfusion unveränderten Blutes sicherere Resultate zu versprechen.

\section{Versuche.}

$\mathrm{Zu}$ diesen Versuchen dienten je zwei Kaninchen von ungefähr gleichem Körpergewicht, von denen jedesmal das kleinere für die Bestimmung der Harnsecretion benutzt wurde, während von dem grösseren das Blut auf das kleine transfundirt wurde.

Der Harn tropfte aus Ureterencantilen in untergestellte Schälchen und wurde in $Z$ wischenräumen von 5 resp. 10 Minuten gemessen. Die Transfusion erfolgte von der Carotis des grösseren in die Jugalaris des kleineren (blutempfangenden, harnsecernirenden) Thieres und dauerte 2-5 Minuten. Die verbindenden Glascanülen waren vorher mit defibrinirtem Blut gefüllt, welches dem grösseren Thiere vorher in geringer Menge von wenigen Cubikcentimetern entnommen war. Füllung der Canülen mit NaCl-Lösung musste vermieden wer-

1) Ponfick, Experiment. Beiträge zur Lehre von der Transfusion. Virch. Archiv Bd. LXII. S. 273. 1875.

2) Lesser, Verhandl. der sächs. Akademie. Math.-phys. Klasse. Bd. XXVI. S. 153.1874 .

3) v. Schröder, Archiv f. experiment. Pathol. u. Pharmakol. Bd. XXII. S. 57.1887 .

4) Schwarz, Ebenda Bd. XLIII. S. 23. 1900. 
den, damit nicht schon dadurch die Blutbeschaffenheit geändert wurde. Die Menge des transfundirten Blutes wurde ermittelt durch sorgfältige Wägung des blutspendenden Thieres vor und nach der Transfusion. Vor der Ueberleitung des Blutes wurde beiden 'Thieren je ein Blutstropfen aus der Carotis zur Hämoglobinbestimmung entnommen, ebenso zu versehiedenen Zeiten nach der Transfusion dem blutempfangenden Thiere, um über den Verbleib des transfundirten Blutes im Versuchsthier Aufschluss zu erhalten (s. u. S. 215).

Zuerst wurden Thiere direct aus dem Stall und in Urethannarkose verwendet. Da jedoch diese Thiere zweifellos versehiedenen Wassergehalt besassen, welcher durch die Resorption der im Magendarmeanal befindlichen 10 procent. Urethanlösung noch gesteigert wurde, und da Gleichheit der Blutbeschaffenheit beider Thiere erstes Erforderniss war, so wurde von Urethanisirung abgesehen und die Thiere nach dem Vorgange v. Limbeck's ${ }^{1}$ ) 2 Tage lang ohne Wasser und Futter gehalten. Dann erhielten sie am 3. Tage je $30 \mathrm{~g}$ pro Kilogramm trocknen Hafers und werden am 4. Tag zum Versuche benutzt. So werden die Thiere auf einen verhältnissmässig niedrigen gleichmässigen Wassergehalt gebracht.

Die Menge des transfundirten Blutes betrug von 33-70 Proc. der Blutmenge des blutempfangenden Thieres. Unangenehme $\mathrm{Zu}$ fälle tratẹn während und nach der Ueberleitung des Blutes nicht auf. Die Thiere ertrugen sie jedesmal sehr gut.

Der Erfolg der Transfusion auf die Harnabsonderung mag durch nachstehende Protokolle veranschaulicht werden:

Versuch II. Kaninchen, $1740 \mathrm{~g}$.

10 h. $45 \mathrm{~m} .-49 \mathrm{~m}$.: Transfusion von $84,5 \mathrm{~g}$ Blut in die Vena jngularis. (Da die ursprüngliche Blutmenge der Thiere [7 Proc.] auf $122 \mathrm{~g}$ zu schätzen ist, beträgt die Vermehrang der Blutmenge durch die Transfusion 69 Proc.).

Harnausscheídung:

$\left.\begin{array}{l}10 \text { h. } 25-35 \mathrm{~m} .: 8 \text { Tropfen } \\ 10 \text { h. } 35-45 \mathrm{~m} .: 5\end{array}\right\}$ Mittel 6,5 Tropfen.

10 h. $45-49$ m.: Transfusion

10 h. $45-55$ m.:

7 Tropfen

10 h. $55-11$ h. 05 m.: 7

11 h. $05-11$ h. $15 \mathrm{~m} .: 6$

11 h. $15-11$ h. 25 m.: 6

11 h. $25-11$ h. 35 m.: 7

Beide Nieren secerniren ganz gleichmässig.

1) v. Limbeck, Archiv f. experiment. Pathol. u. Pharmakol. Bd. XXVIII. S. 70.1889 . 
Man sieht, dass durch eine ganz beträchtliche Vermehrung der Blutmenge eine Zunahme der Harnabsonderung nicht erfolgte.

Ein etwas anderes Resultat gab gleich der nächste Versuch:

Versuch III. Kaninchen. $1490 \mathrm{~g}$.

10 h. $50-55$ m.: Transfusion von $72,5 \mathrm{~g}$ Blut (= 70 Proc. der ursprünglichen Blutmenge) in die Vena jugularis.

Harnauss cheidung:

10 h. $20-10$ h. 30 m.: 4 Tropfen

10 h. $30-10$ h. $40 \mathrm{~m} .: 7=$

10 h. $40-10$ h. $50 \mathrm{~m} .: 4=$

10 b. $50-10$ h. $55 \mathrm{~m}$. Transfusion

10 h. $50-11$ h. - m.: 14 Tropfen

11 h. -11 h. $10 \mathrm{m.}: 12$

11 h. $10-11$ h. $20 \mathrm{~m} .: 4$ =

11 h. $20-11$ h. $30 \mathrm{~m} .: 5$ =

$11 \mathrm{~h}, 30-11 \mathrm{~h}, 40 \mathrm{~m} .: 5=$

Hier hat eine deutliche, aber sehr geringe Steigerung der Harnabsonderung stattgefunden. Es wurde in 20 Minuten ein Plus von 18 Tropfen, also weniger als $1 \mathrm{ccm}$, beobachtet. Dieses ist die grössteZunahme des Harnflusses, welche in allen unseren Versuehen vorkam. Es tritt also nach Transfusion möglichst gleichartigen Blutes entweder gar keine oder nur eine ganz minimale Steigerung der Urinsecretion auf, welche sich in unseren Experimenten immer unterhalb des Werthes von insgesammt 1 com hielt.

Dasselbe gilt für Hunde:

Versuch X. 2 Hunde hungern und dursten $3 \times 24$ Stunden und erhalten 1 Stunde vor Beginn des Versuches je $6 \mathrm{mg}$ pro Kilogramm Morphin. mur. subcutan. Sonstige Versuchsanordnung wie bei den Kaninchen.

$\mathrm{Hund}, 2800 \mathrm{~g}$.

10 h. $55-571 / 2$ m.: Transfusion von $164 \mathrm{~g}$ Blut (=84 Proc. der ursprünglichen Blutmenge) in die Vena jugularis.

Harnausscheidung:

10 h. $35 \mathrm{~m} .-10$ h. $45 \mathrm{~m} .: 0,2 \mathrm{ccm}$

10 h. 45 m. -10 h. 55 m.: 0,2 =

10 h. $55 \mathrm{~m}$. -10 h. $571 / 2$ : Transfusion

10 h. 55 m. -11 h. 05 m.: $0,25 \mathrm{ccm}$

11 h. 05 m. -11 h. 15 m.: $0,25=$

11 h. $15 \mathrm{~m} .-11$ h. $25 \mathrm{~m} .: 0,25$ =

Also auch hier keine irgendwie deutliche Zunahme der Harnmenge.

Es erhebt sich nun zunäcbst eine Frage, deren Entseheidung für den Werth der Versuche von ausschlaggebender Bedeutung sein 
musste: ob nämlich durch die Transfusion eine wirkliche Zunahme der Blutmenge erzielt werde, ob der Austritt von Flüssigkeit aus dem Blut ins Gewebe, welcher bekanntlich ${ }^{1}$ ) nach jeder 'Transfusion statthat, nicht so gross sei, dass dabei eine wirkliche Plethora überhaupt nicht zu Stande komme.

Ueber die Aenderung der Menge des Blutes ldurch und nach der Transfusion suchte ich durch Ilb-Bestimmungen bei beiden Versuchsthieren vor und bei dem blutempfangenden Thiere nach der Blutüberleitung Aufschluss zu bekommen. Es wurde wieder das Miescher'sche Hämoglobinometer ${ }^{2}$ ) benutzt. Für die Berechnung wurde wie in den früheren Arbeiten die ursprüngliche Blutmenge des Thieres zu 7 Proc. des Körpergewichtes angenommen. Es war aber noch eine zweite Voraussetzung, die bei den Experimenten erfüllt sein musste. Es durfte, um eine Berechnung möglich zu machen, ein Austritt von rothen Blutkörperehen aus der Gefässbahn nicht stattgefunden haben. Dieses liess sich in der That niemals nachweisen. Stets überzeugte ich mich nach Sehluss des Versuches durch die Section, dass rothgefärbte Ergtisse in die serösen Höblen fehlten. Es wurde vielmehr Brust- und Bauchhöhle stets trocken gefunden. Ekchymosen u. s. w. waren niemals zu sehen. Auch die Blutaustritte in den Darm, welche $\mathrm{Regéczy^{3 }}$ ) beschreibt, wurden in keinem Falle gefunden. Dieses vorausgeschickt, liess sich die Berechnung nun folgendermaassen ausfübren:

Versuch II. Kaninchen, $1740 \mathrm{~g}$, also Blatmenge $122 \mathrm{~g}$. Hb-Gehalt $=15,38$ Proc.

Hb-Gehalt des transfundirten Blutes $(84,5 \mathrm{~g})=15,12$ Proc. 122 ursprüngliches Blut enthalten $18,76 \mathrm{~g} \mathrm{Hb}$

Summa $\frac{84,5 \text { Transfusionsblut }}{206,5 \text { Blut }} \quad$ mit $31,54 \mathrm{~g} \mathrm{Hb}$

Da nun aber 20 Minuten nach der Transfusion der $\mathrm{Hb}$-Gehalt des Blutes $=19,78$ Proc. gefunden wurde, so sind die $31,54 \mathrm{~g} \mathrm{Hb}$ nicht in 206,5 , sondern in nur $159,5 \mathrm{~g}$ Blut enthalten. Es wird also 206,5-159,5 $=47 \mathrm{~g}$ Flüssigkeit in die Gewebe treten. Das sind bereits 56 Proc. des transfundirten Blutes, resp. 22 Proc. des Gesammtblutes (ursprünglichen + transfundirten Blutes). Die Zunahme der Blutmenge beträgt also nur noch $84,5-47=37,5 \mathrm{~g}$. Es ergiebt sich eine Zunahme der Blutmenge von $100: 131$.

Nach diesen Berechnungen seien in Folgendem einige der erhaltenen Resultate zusammengestellt:

1) Lesser, a. a. O. - v. Regé czy, Pflüger's Arch. Bd.XXXVII. S. 73. 1885. S. $431 \mathrm{f}$.

2) Vgl. II. Mittheilung. Archiv f. exper. Pathol. u. Pharmakol. Bd. XLIV.

3) v. Regéczy, a. a. 0 . 


\begin{tabular}{|c|c|c|c|c|c|c|c|c|c|}
\hline \multirow{2}{*}{ Kaninchen } & \multirow{2}{*}{$\begin{array}{c}\begin{array}{c}\text { Versuchs- } \\
\mathrm{Nr} .\end{array} \\
\text { II }\end{array}$} & \multirow[t]{2}{*}{$\begin{array}{l}\text { Zeit nacb } \\
\text { Beginn } \\
\text { der Trans- } \\
\text { fusion }\end{array}$} & \multicolumn{2}{|c|}{$\begin{array}{l}\text { Trans- } \\
\text { fusions- } \\
\text { menge }{ }^{1} \text { ) }\end{array}$} & $\begin{array}{c}\text { Blut- } \\
\text { mengen- } \\
\text { zanabme }\end{array}$ & \multicolumn{4}{|c|}{$\begin{array}{l}\text { Flussigkeitsaustritt aus } \\
\text { der Gefässbabn in Proc. der } \\
\text { Transfusions- Gesammt- } \\
\text { mengc } \\
\end{array}$} \\
\hline & & & 69 & Proc. & 131 & 56 & Proc. & 22 & Proc. \\
\hline$=$ & III & $10^{\prime}$ & 70 & $=$ & 150 & 28 & $=$ & 11 & $=$ \\
\hline \multirow[t]{2}{*}{$=$} & IV & $4^{\prime}$ & 33 & $=$ & $\{21$ & 38 & $=$ & 9 & $=$ \\
\hline & IV & $65^{\prime}$ & 33 & $=$ & 117 & 50 & $=$ & 12 & $=$ \\
\hline Kaninchen & $\mathrm{V}$ & $6^{\prime}$ & 41 & $=$ & 134 & 19 & $=$ & 5 & $=$ \\
\hline Hund & VI & $8^{\prime}$ & 88 & $=$ & 171 & 19 & $=$ & 9 & $=$ \\
\hline$=$ & VII & 7 & 132 & $=$ & 195 & 28 & $=$ & 16 & $=$ \\
\hline \multirow{3}{*}{$\begin{array}{l}= \\
\text { Mittel: }\end{array}$} & $\mathrm{X}$ & $3^{\prime}$ & 84 & $=$ & 149 & 41 & $=$ & 19 & $=$ \\
\hline & & & & & $\overline{146}$ & & & & \\
\hline & & I & I & II & III & & IV & 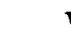 & $V$ \\
\hline
\end{tabular}

Man sieht aus dieser Tabelle, dass Bb-Bestimmungen zu den verschiedensten Zeiten nach der Transfusion (von 3 Minuten bis zu einer Stunde) angestellt wurden. Zu allen diesen Zeiten war die Blutmenge des Thieres deutlich gesteigert, und zwar war die kleinste Steigerung von 100 auf 117 (nach 65 Minuten), die grösste - beim Hund - von 100 auf 195 (nach 7 Minuten). Die mittlere Zunahme der Blutmenge betrug 146. Berücksichtigt man, dass bei starken Diuresen nach Salzwasserinfusion (II. Mitth. S. 410) die Blutverdünnung im Mittel nur 117 betragen hatte, so ergiebt sich, dass bei den hier geschilderten Transfusionsversuchen wirklich eine Zunahme der Blutmenge statthatte, welche zur Diurese hätte führen können.

Trotzdem tritt bei Bluttransfusion bei einer mittleren Zunahme der Blutmenge auf 146 keine Diurese, nach Salzwasserinfusion bei einer Blutmengensteigerung auf 117 starke Harnfluth auf. Man sieht schon hieraus, dass die Plethora an sich für das Zustandekommen der Diurese nicht verantwortlich gemacht werden kann.

Die Tabelle zeigt aber weiter, dass durchaus nicht alles Blut in der Blutbahn geblieben ist, sondern, wie schon Lesser und Regéczy angeben, zum Theil die Gefässe verlassen hat. So wurde z. B. in Versuch II, in dem die Transfusionsmenge 69 Proc. der ursprünglichen Blutmenge betrug, die Zunahme der Flüssigkeit in der Blutbahn statt 169 nur 131 gefunden. Es muss also Flüssigkeit aus der Blutbahn in die Gewebe getreten sein. Wie sich dieser Aus-

1) Menge des transfundirten Blutes in Proc. der ursprünglichen Blutmenge des Versuchsthieres.

2) Noch bestehende Zunahme der ursprünglichen Blutmenge im Moment der zweiten Hb-Bestimmung $\mathrm{nach}$ der Transfusion bezogen auf 100 . 
tritt quantitativ verhält, lehrt Columne V: Es sind zu den verschiedenen Zeiten von der nach Schluss der Transfusion im Gefässsystem kreisenden Gesammtblutmenge 5-22 Proc. Flüssigkeit in die Gewebe getreten. Die Grösse dieses Flüssigkeitsaustrittes steigt mit der Zeit, welche nach der Transfusion verstreicht. Versuch II und III lassen sich zu diesem Zwecke vergleichen, weil die Menge des transfundirten Blutes in beiden Fällen so gut wie gleich war. Nach 10 Minuten haben 11 Proc., nach 20 Minuten 22 Proc. die Gefässbahn verlassen. In Versuch IV wurde die Blutmenge zu zwei Zeiten bestimmt. Der Austritt betrug nach 4 Minuten.9 Proe. und nach 65 Minuten 12 Proc.

Je grösser die Menge des transfundirten Blutes ist, um so länger dauert der Flüssigkeitsaustritt aus den Gefässen an. In Versuch II und III war die Blutzufuhr eine beträchtliche. Deshalb steigt in den zweiten 10 Minuten die austretende Menge noch von 11 Proc. auf 22 Proc. In Versuch IV dagegen wurde nur wenig Blut transfundirt. Hier hat der hauptsächliche Flüssigkeitsaustritt bereits in den ersten 4 Minuten stattgefunden, und in weiteren 61 Minuten steigt diese ausgetretene Menge nur von 9 auf 12 Proc.

Col. IV zeigt nun den Effect dieses Stromes aus dem Blut ins Gewebe. Von dem transfundirten Blute ist zur Zeit der Hb-Bestimmungen schon immer ein beträchtlicher Theil aus der Blutbalun entfernt (19-56 Proc.). In Versuch IV haben nach 65 Minuten 50 Proc., in Versuch II nach 20 Minuten sogar 56 Proc. des eingeführten Blutes die Gefässbahn verlassen. Es ist also der grössere Theil des Plasmas ausgetreten. Die Bestimmungen, welche kiurzere Zeit nach der Transfusion ausgeführt wurden, zeigen, dass der Verlust von Flüssigkeit an die Gewebe ein geringerer ist.

Es lehren also diese Zahlen, dass unmittelbar nach einer Vermebrung der Blutmenge, bei welcher sich die Beschaffenheit des Blutes nicht ändert, ein lebhafter Austritt von Flüssigkeit aus den Gefässen statthat, dass der grösste Theil dieses Austrittes in die ersten Minuten nach der Blutzufuhr fällt, und dass je nach der Grösse der Transfusion dieser Strom in die Gewebe schneller oder langsamer abnimmt. Nach 20-60 Minuten ist der grössere Theil des zugeführten Plasmas in die Gewebe gegangen, während der kleinere Theil mit den rothen Blutkörperchen in der Gefässbahn verbleibt. Ihr ferneres Sobioksal hier zu untersuchen, liegt nicht im Plan dieser Arbeit.

Da das zugeführte Blut dem ursprïnglichen so gut wie gleichartig ist, so kann für diesen Flüssigkeitsaustritt wohl kaum Diffusion und Osmose verantwortlich gemacht werden. Auch für eine ge- 
steigerte secretorisehe Thätigkeit der Capillarzellen liegen gar keine Gründe vor. Wir müssen deshalb für die Beseitigung des Flüssigkeitsüberschusses Filtration verantwortlich machen. Erstaunlich ist nun, mit welcher Promptheit diese Filtration eintritt und in wenigen Minuten bereits grosse Theile des Zugeführten wieder beseitigt. Dieser Flüssigkeitsaustritt findet dabei also nur durch die Körpercapillaren, nicht durch die Nierengefässe statt. Es zeigt sich demnach auch hier wieder, dass die Flüssigkeitsausscheidung in den Nieren nicht denselben Bedingungen unterworfen sein kann, wie jene aus den Capillarbezirken des übrigen Körpers.

Nachdem auf die geschilderte Weise festgestellt war, dass durch die benutzte Methode der Transfusion wirklich eine Zunahme der Blutmenge erzielt wurde, welche grösser ist, als sie in den frïheren Versuchen mit Salzwasserinfusion eintrat, war nunmehr zu untersuchen, ob sich auch die Kreislaufsverhältnisse ähnlich gestalten. Zunächst wurde das Verhalten des arteriellen und venösen Druckes geprüft. Hierzu wurden Hunde benutzt, welche $3 \times 24$ Stunden gehungert und gedurstet hatten und mit $6 \mathrm{mg}$ pro Kilogramm Morphin. mur, narkotisirt waren. Zur Druckmessung dienten Art. und Vena femoralis. Die Versuchsanordnung war die gleiche, wie sie in der II. Mittheilung geschildert wurde (S. 420). Das Resultat eines derartigen Experimentes wird durch nebenstehende Curve veranschaulicht.

Man sieht, dass alsbald mit Beginn der Transfusion sich der arterielle Blutdruck beträehtlich (von 96 auf $152 \mathrm{~mm} \mathrm{Hg}$ ) hebt und sich nach Schluss derselben wieder auf etwa $130 \mathrm{~mm}$ senkt, um auf dieser Höhe zu bleiben. Der venöse Druck, dessen Werthe in Höhen der $25 \mathrm{Proc} . \mathrm{MgSO}_{4}-\mathrm{Säu} \mathrm{le}$ angegeben sind, steigt ebenfalls rapide an, von 64 auf $165 \mathrm{~mm} \mathrm{Mg}$-Lösung, um dann langsam zu sinken. Auch er ist aber bis zum Schluss des Versuches dauernd stark gesteigert. Die Diurese (in Cubikcentimeter pro Kilogramm Thier und 10 Minuten) dagegen zeigt keine Spur von Zunahme. 1) - Das Ver-

1) Dieser Versuch ist insofern als besonders gelungen zu bezeichnen, als dabei stärkere pulsatorische und exspitatorische Schwankungen des Blutdruckes nicht auftraten. In anderen Experimenten gelangte bei derartigen Druckänderungen leicht etwas von der Mg-Lösung, mit der die Manometer gefüllt waren, in die Blutbahn des Thieres und genügte, um eine dentliche Zunahme der Harnsecretion zu bewirken, da ja die Normalsecretion beim Hungerthier eine ganz geringe ist und jede Steigerung sich sofort deutlich bemerkbar macht. Deshalb wurden in einzelne Versuchen zur Füllung de Venenmanometers defibrinirtes Blut verwendet. Aber auch dann liess sich der geschilderte Missstand manchmal nicht 
halten des Venendruekes war insofern in diesen Versuchen etwas atypisch, als er noch nach Schluss der Transfusion weiter stieg. In den anderen Fällen sank er gleich nach Schlluss der Blutzufuhr ab. Sonst aber wurde stets das gleiche Ergebniss gewonnen; arterieller und venöser Druck stiegen stark an und blieben auch nach Schluss der Transfusion während der Dauer der Beobachtung (meist 20 Min.)

Versuch VI.

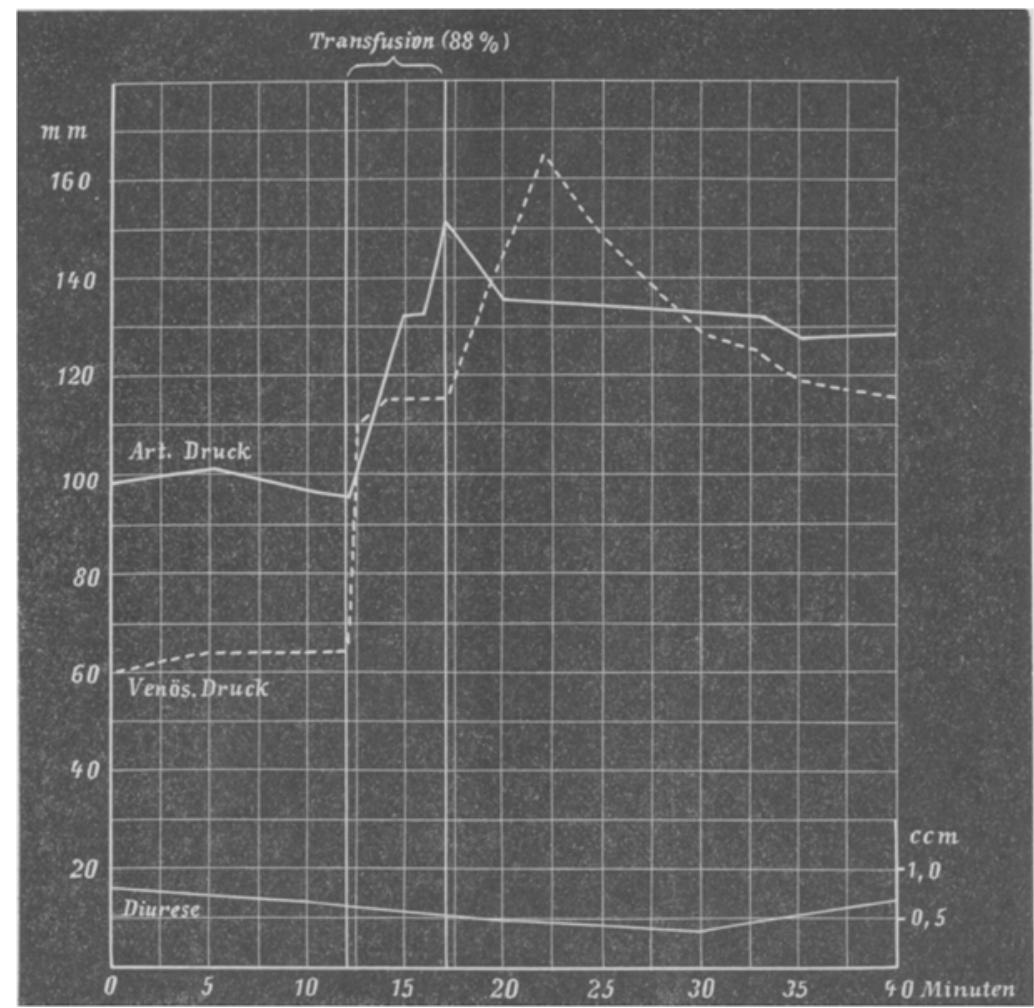

deutlich erhöht. Die Zunahme des arteriellen Druckes betrug am Schluss der Versuche meist noch $30-40 \mathrm{~mm}$, der Venendruck war gewöhnlich noch auf das Doppelte gesteigert.

Diese Erhöhung des arteriellen sowohl wie des venösen Druckes muss nun nach Starling ${ }^{1}$ ) auch eine Zunahme des allgemeinen

vermeiden, da das gleiche am Arterienmanometer auftrat. Es mussten deshalb an einer Reibe von Thieren die Druckverbältnisse obne Berücksichtigung der Harnsecretion fest gestellt werden.

1) Starling, Journal of physiol. Vol. XVI. S. 159. 1894. 
Capillardruckes zur Folge haben. Trotz dieser Drucksteigerung in den Capillaren ist aber keine Diurese aufgetreten.

Es fragte sich nur noch, ob denn auch wirklich in den Nieren eine solehe capillare Drucksteigerung stattfände. Es wäre nämlich möglich, dass sie durch active Vasoconstriction der kleinsten Nierenarterien übercompensirt würde und deshalb in den Glomerulis nicht in Wirksamkeit treten konnte. Hierüber musste das Onkometer Aufschluss geben.

Versuch XIV.1)

Hund, 4330 g, Morphinnarkose.

Transfusion 70 Proc.

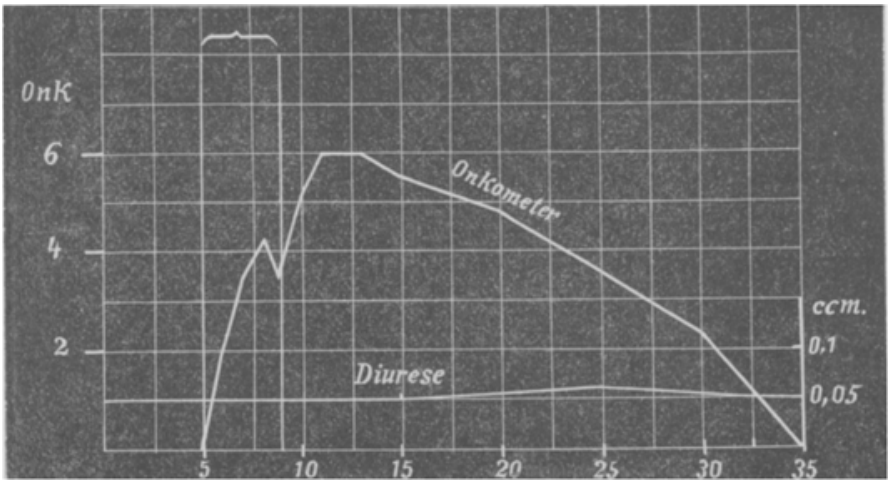

Die Curve zeigt, dass die Diurese (registrirt in Cubikcentimeter pro Kilogramm Thier in 10 Minuten) durch die Transfusion ungeänder't bleibt, dass dagegen das Onkometer alsbald ansteigt und eine Vergrösserung des Nierenvolumens bis 6 Proc. anzeigt, die dann langsam wieder zurückgeht. Ein ähnliches Verhalten wurde in weiteren Experimenten an Kaninchen und Hunden festgestellt; hier nahm z. B. das Nierenvolumen um 7 und 9 Proc. zu. Dieses sind Ausschläge, wie sie bei der Salzdiurese ebenfalls beobachtet werden (vgl. II. Mittheilung S. 424). Es beweist diese Volumenszunahme der Niere, dass die Steigerung des allgemeinen Capillardruckes auch in der Niere evident wird, dass eine Vasoconstriction bei derartigen Transfusionen in der Niere nicht vorkommt. Dagegen kann man natürlich aus dem Onkometerausschlag anf eine active Vasodilatation in

1) In den Onkometerversuchen wurde von gleichzeitiger Registrirung des arteriellen Druckes Abstand genommen, weil sein Verhalten aus den vorher geschilderten Experimenten bekannt war und das oben geschilderte Rüclssteigen der Mg-Lösung aus dem Manometer in die Blutbahn hier natürlich den ganzen Versuch verdorben bätte. 
der Niere nicht schliessen, da ja gleichzeitig der allgemeine arterielle und renöse Druck steigen. Das Onkometer zeigt nur an, dass sich die Niere an der allgemeinen Drucksteigerung im Körper betheiligt.

Wir sehen also, dass nach Transfusion eine deutliche und hochgradige Steigerung des allgemeinen arteriellen, venösen und capillaren Druckes und eine Volumenszunahme der Niere eintritt, und dass trotzdem eine wesentliche Zunahme der abgeschiedenen Harnmenge nicht statthat.

Kebren wir zum Ausgangspunkt unserer Betrachtungen zurück, so ergiebt sich auf die Anfangs aufgeworfenen Fragen die Antwort, dass, wenn jede Aenderung der Blutzusammensetzung vermieden wird, eine Zunahme der Blutmenge (Plethora) nicht genügt, um eine irgendwie bedeutendere Diurese herbeizuführen. Eine Zunahme der abgeschiedenen Harnmenge bleibt entweder ganz aus oder ist so minimal, dass sie mit der bei der Salzdiurese beobachteten nicht verglichen werden kann. Diese Versuche bekräftigen also die Anschauung, dass wir in der Aenderang der Blutzusammensetzung die eigentliche Ursacbe der Salzdiurese zu sehen haben, und zeigen weiter, dass der Einfluss der Kreislaufsänderungen nur ein ganz minimaler sein kann. Es ist hier gewissermaassen die Gegenprobe auf die in der II. Mittheilung gezogenen Folgerungen gemacht.

Es liegt mir natürlich fern, durch diese Untersuchungen jeden Einfluss der Circulation auf die normale Harnabscheidung, welche durch zahlreiche Arbeiten des Ladwig'schen Laboratoriums und Anderer festgestellt wurde, leugnen zu wollen. Sie weisen aber darauf hin, dass man den Einfluss capillarer Drucksteigerung in den Glomerulis nicht tuberschätzen darf. $\mathrm{Es}$ ist tuberraschend, als wie geringfügig sich die Beeinflussung der Urinsecretion durch die geschilderten Kreislaufsänderungen gezeigt hat.

Es erübrigt nun noch, nachzuweisen, dass, wenn man Versuchsbedingungen schafft, unter denen durch die Transfusion ein e Veränderung der Blutbeschaffenheit erzielt wird, dass dann eine Steigerung der Harnmenge eintritt. Zu diesem Zwecke wurde dem blutspendenden Thiere vor dem Versuche eine Infusion von Glaubersalzlösung gemacht und dann erst, nachdem hierdarch eine Aenderung der Blutzusammensetzung erzielt war, die Ueberleitung auf das zweite Thier vorgenommen. Berücksichtigt man, dass durch intravenöse Zufuhr von 7,8 Proc. Glaubersalzlösung unter den in der II. Mittheilung eingehaltenen Versuchsbedingungen der Glaubersalzgehalt des Blutes auf $0,3-0,4$ Proc. steigt, dass dann dieses Blut 
einem $z w e i t e n$ Thiere transfundirt wird und dann wieder eine Veltheilung dieser geringen Salzmenge zwischen Blut und Gewebe statthat, so wird die Glaubersalzzunahme, welche auf die Niere des 2. Thieres zur Wirknng gelangen kann, nur eine ganz geringe sein, und es war daher a priori nicht ausgeschlossen, dass sich ein Einfluss überhaupt nicht constatiren lasse. Trotzdem war derselbe deutlich

Versuch XV. Einem Kaninchen von $1770 \mathrm{~g}$ wird 46 Minuten lang $\mathrm{Na}_{2} \mathrm{SO}_{4}$ in 7,85 procent. Lösung mit einer Einlaufsgeschwindigkeit von $0,6 \mathrm{ccm}$ pro Minute und Kilogramm intravenös eingeführt. Nach Schluss des Einlaufs werden $54 \mathrm{~g}$ Blut dieses Thieres auf ein zweites Kaninchen von $1712 \mathrm{~g}$ transfundirt (Transfusionsmenge 45 Proc.).

Harnausscheidung:

3 h. $45 \mathrm{~m} .-3$ h. $55 \mathrm{~m} .: 0,45 \mathrm{ccm}$

3 h. $55 \mathrm{~m} .-4$ h. $05 \mathrm{~m} .: 0,2=$

4 h. $06 \mathrm{~m} .-4$ h. $08 \mathrm{~m}$. : Transfusion

4 h. $05 \mathrm{~m} .-4$ h. $15 \mathrm{~m} .: 1,2 \mathrm{ecm}$

4 h. $15 \mathrm{~m} .-4$ b. $25 \mathrm{~m} .: 1,05=$

4 h. $25 \mathrm{~m} .-4$ h. $35 \mathrm{~m} .: 0,9$,

Man sieht, dass die Harnmenge ganz deutlich zunimmt und zwar so beträchtlich, wie das nach einfacher Transfusion nie beobàchtet wurde.

$\mathrm{Ja}$, in einem anderen Versuche, wo die Versehiedenheit der Blutzusammensetzung nur dadureh bewirkt wurde, dass das blutspendende Thier dureh zweitägiges Fasten und Darreichung von trockenem Futter am 3. Tage wasserarm gemacht war, während das blutempfangende Thier reichliches feuchtes Futter erhalten hatte, liess sich gleichfalls eine Wirkung auf die Harnmenge constatiren. Das ausgeschiedene Urinquantum stieg anf das $3-4$ fache.

Es wird also die Transfusion sofort anf die Nierenthätigkeit wirksam, sowie sie zu einer Aenderung der Blutbeschaffenheit fübrt.

\section{Zusammenfassung.}

1. Durch Transfusion gelingt es, ohne Aenderung der Bhutzusammensetzung starke Plethora mit Steigerung des arteriellen, venösen und capillaren Druckes nnd Volumenszunahme der Niere zu erzeugen.

2. Hierbei kommt es zu reichlichem Austritt von Flüssigkeit aus den Gefässen in die Gewebe. Dagegen tritt keine Diurese ein.

3. Schafft man Bedingungen, unter denen bei Transfusion eine Aenderung der Blutbeschaffenheit eintritt, so steigt die Harnauscheidung.

4. Daraus folgt, dass die Plethara mit ihren Folgezuständen die Crsache der Salzdiurese nicht sein kann, sondern dass diese in der Aenderung der Blutzusammensetzung gesehen werden muss. 